\title{
Role of Micronucleus in Cervical Intraepithelial Lesions and Carcinoma
}

\author{
Suganya Kuppovi Reddy ${ }^{1 *}$, Surendra Kumar Verma ${ }^{1}$, Sajini Elizabeth Jacob ${ }^{1}$, \\ Neelaiah Siddaraju' ${ }^{1}$, Dasari Papa ${ }^{1}$, Suthanthira Kannan ${ }^{2}$ \\ ${ }^{1}$ JIPMER, Puducherry, India \\ ${ }^{2}$ Government Medical College, Kozhikode, India
}

\begin{abstract}
Background: Micronuclei (MN) are considered sensitive indicators of chromosomal damage. Studies have emphasized the utility of MNscoring in detection of increased risk of various cancers in humans. More recent studies have shown MN assay to be highly promising in cervical cancer screening.

Aims: To evaluate the utility of micronuclei in distinguishing various squamous intraepithelial lesions and invasive squamous cell carcinoma of cervix.

Methods: In this descriptive study, a total of 100 cases were studied, of which 50 were normal (control), 10 were high grade squamous intra epithelial lesions (HSIL) and 26 were invasive squamous cell carcinomas (SCC). Remaining 14 cases were equivocal entities comprising 3 ASCUS and 11 ASC-H cases. In each case, MN-count was done per 1000 epithelial cells and a consensus MN-score was taken after a consensus and careful review by 4 cytopathologists.

Results: The mean MN-score showed a gradual increase from normal smear to invasive SCC cases. MN-score of HSIL and invasive SCC were significantly higher than the normal and ASC-H smears. Cases with premalignant/ malignant outcome had a significantly high MNscore as compared to cases with reactive outcome.

Conclusion: MN-scoring is a simple, non invasive and cost effective test, which can be performed on an easily collected exfoliated cell sample. MN-assay in conjunction with conventional Pap test can be an effective tool for screening cervical cancer and identifying women at risk.
\end{abstract}

Keywords: Micronuclei, MN-score, Cervical Cancer, Precancerous Lesions

\section{Introduction}

Micronucleus (MN) originates from chromosome fragments or whole chromosomes that are not included in the main daughter nuclei during nuclear division. ${ }^{[1]}$ Its presence in a cell is a sensitive indicator of chromosomal damage. A direct association between increased $\mathrm{MN}$ frequency and cancer development has already been reported in head and neck, ${ }^{[2]}$ colonic, ${ }^{[3]}$ cervical, ${ }^{[4]}$ and urothelial malignancies. ${ }^{[5]} \mathrm{MN}$ frequency is also increased in certain chromosome breakage syndromes such as Bloom syndrome and ataxia telangiectasia,as well as, in persons exposed to chemical carcinogens and ionizing radiation, indicating the increased risk of cancer. ${ }^{[6]}$

Cervical cancer is one of the most common cancers in India and Papanicolaou (Pap) cervical cytology is an effective screening method for its early detection. Bethesda 2001 system for reporting cervical cytology has certain equivocal entities like Atypical Squamous Cells of Undetermined Significance (ASCUS) and Atypical Squamous Cells cannot exclude HSIL (ASC-H) ${ }^{[7]}$ Only a minor proportion of cases reported as ASCUS and ASC-H turn out to be cervical intraepithelial lesion (CIN) on histopathology. ${ }^{[8,9]}$ HPV DNA testing is a useful adjunct in identifying those high risk cases, but it has cost limitations. ${ }^{[8]}$ Therefore, there is a need for a simple, reliable, reproducible \& cost effective method for identifying the true CIN cases, among those reported as ASCUS and ASC-H.

Over the last decade, the wide applicability of the micronucleus test in peripheral blood lymphocytes and the simplicity of scoring has made it an attractive cytogenetic tool to assess occupational and environmental exposures to genotoxic agents. Micronucleus counting in cervical lesions, a simple non invasive procedure, helps to stratify pre-invasive lesions. Thus, this method in conjunction with present cytology-based test will be a helpful tool for triage, follow up or treatment of borderline or doubtful cases. ${ }^{[10]}$ 
Aims and Objectives: To evaluate the utility of micronuclei in distinguishing various squamous intraepithelial lesions and invasive squamous cell carcinoma of cervix

\section{Materials and Methods}

Our study is a descriptive study conducted in the Department of Pathology and Obstetrics \& Gynaecology, JIPMER between January 2012 and March 2013. In this we compared the micronucleus scoring in the whole spectrum of cervical lesions.

A total of 100 cases interpreted as per the Bethesda 2001 system for reporting cervical cytopathology between January 2012 and June 2013 were examined. Among them 50 were normal smears without any intraepithelial abnormalities, 10 were premalignant lesions- high grade squamous intra epithelial lesions (HSIL) and 26 were frank invasive squamous cell carcinomas. Remaining 14 cases were equivocal entities including 3 cases of ASCUS and 11 cases of ASC-H.

Smears with (i) scant cellularity, (ii) marked drying artifact, (iii) squamous cells obscured by intense inflammation and hemorrhage and (iv) poor staining were excluded from the study.

These smears were subjected to routine Papanicolaou staining after fixation with $95 \%$ ethanol in the cytology laboratory. Final interpretation of all the cases was made after careful evaluation by four different cytopathologists. MN-frequency was studied with strict criteria in all the cases by counting 1000 squamous epithelial cells under oil immersion magnification (Figure $1 \& 2$ ).

Criteria for Micronuclei: The criteria for designating an extra-nuclear body as "micronucleus" were: ${ }^{[1,2,7]}$

1. Diameter $1 / 16$ to $1 / 3$ of the main nucleus

2 Staining intensity similar to, or slightly weaker than, that of the nucleus

3. Round-to-oval shape with a smooth perimeter i.e. the borders should be distinctly recognizable indicating the presence of a nuclear membrane

4. Color and texture same as that of the main nucleus

5. Close proximity, but no actual contact with the nucleus i.e. absence of overlap with or bridge to the nucleus

6. Plane of focus same as that of the main nucleus

7. Non refractile

A consensus 'micronucleus frequency' was taken after the smears were evaluated by four cytopathologists independently. Cells with double or multiple $\mathrm{MN}$ were also given a score of one. Keratohyaline granules, candida, stain deposits, bacterial colonies, cytoplasmic fragments, blood elements, sperms, apoptotic bodies, karyorrhetic material were carefully looked for and exempted from counting (Figure 3).

Frequency of MN in various squamous intra-epithelial/ invasive lesions was compared among the various groups including the control group. Pre-biopsy cytologic interpretation and micronucleus frequency was compared with the histopathologic diagnosis.

\section{Results}

The mean age of the patients in normal, ASCUS, ASC-H, HSIL, SCC of cervical lesions were $38.52 \pm 12.49$, $45.67 \pm 15.63, \quad 44.36 \pm 8.80, \quad 40.90 \pm 9.25, \quad 56 \pm 9.89$ years respectively (Table 1). The mean age was more in patients with invasive SCC than in normal and precursor lesions. The mean MN-score in normal smears andvarious cervical lesions and its trend are shown in table-1 and figure-4; while, biopsy outcome of the patients with cervical lesions are shown in table-2.Biopsy was not available for control cases (normal smears)

There was a gradual and stepwise increase in $\mathrm{MN}$ score from normal to ASCUS to ASC-H to HSIL and to invasive SCC. Analysis of variance(ANOVA) was applied to analyse the difference in mean values of $\mathrm{MN}$-scores among different groups. The increase in $\mathrm{MN}$-score was more significant in invasive SCC group as compared to control and ASC-H groups, but the MN- score between the HSIL and invasive SCC group was not significant though it showed a gradual increase.

We also correlated the $\mathrm{MN}$-score with final biopsy outcome (Table-3). We noted a progressive increase in mean MN score from non-malignant to malignant cases. Two or more $\mathrm{MN}$ in a single cell wasa rare feature and was noted in Invasive SCC.

Taking biopsy diagnosis as the gold standard, sensitivity, specificity, positive predictive value (PPV) and negative predictive value (NPV) of MN was calculated. MN were found to be $100 \%$ sensitive and $72 \%$ specific in detecting squamous intraepithelial lesions and SCC with a PPV and NPV of $95 \%$ and $100 \%$ respectively.

\section{Discussion}

Cervical cancer is one of the most common cancers in India $^{[10]}$. The phenotypic changes of cervical cancer are always preceded by genetic damage caused by various carcinogens. Human papillomavirus (HPV) is a causal factor in the development of cervical cancer and its precursor lesion, cervical intraepithelial neoplasia (CIN). 
Table 1: Age-distribution and micronucleus frequency in cervical lesions.

\begin{tabular}{|l|c|c|c|}
\hline Group & $\begin{array}{c}\text { No } \\
\text { of Cases }\end{array}$ & $\begin{array}{c}\text { Mean age } \pm \text { Standard deviation } \\
\text { (years) }\end{array}$ & $\begin{array}{c}\text { Mean MN-Score } \pm \text { Standard } \\
\text { deviation }\end{array}$ \\
\hline Normal & 50 & $38.52 \pm 12.49$ & $0.04 \pm 0.19$ \\
\hline ASCUS & 3 & $45.67 \pm 15.63$ & $1.67 \pm 1.53$ \\
\hline ASC-H & 11 & $44.36 \pm 8.80$ & $5.27 \pm 5.36$ \\
\hline HSIL & 10 & $40.90 \pm 9.25$ & $9.5 \pm 2.95$ \\
\hline SCC & 26 & $56.00 \pm 9.89$ & $10.77 \pm 3.96$ \\
\hline
\end{tabular}

Table 2: Biopsy outcome of various cervical lesions.

\begin{tabular}{|l|c|c|c|c|c|c|}
\hline \multirow{2}{*}{ Groups } & \multirow{2}{*}{$\begin{array}{c}\text { No of } \\
\text { cases }\end{array}$} & \multicolumn{5}{|c|}{ Biopsy outcome } \\
\cline { 4 - 7 } & Normal & Chronic cervicitis & Metaplasia & Premalignant (CIN) & Malignant \\
\hline ASCUS & 3 & 1 & 1 & 1 & 1 & 5 \\
\hline ASC-H & 11 & 3 & - & - & 7 & 2 \\
\hline HSIL & 10 & - & - & - & 2 & 3 \\
\hline SCC & 26 & - & - & & 24 \\
\hline
\end{tabular}

Table 3: Correlation of MN frequency with final biopsy outcome.

\begin{tabular}{|l|c|c|c|}
\hline Biopsy outcome & No of cases & MeanMN-Score \pm Standard deviation & P-value \\
\hline Non malignant & 7 & $0.71 \pm 1.25$ & $\mathrm{P}<0.001$ \\
\hline Pre malignant & 14 & $7.93 \pm 3.38$ & \\
\hline Malignant & 29 & $11.10 \pm 3.68$ & \\
\hline
\end{tabular}

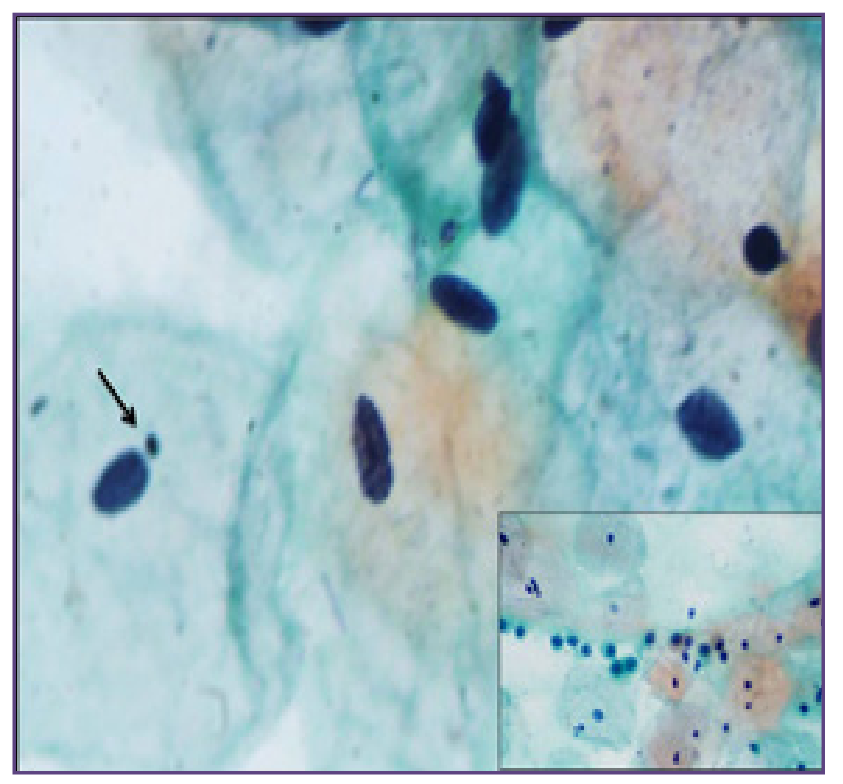

Fig. 1: MN in the cervical smear of HSILcase.(Pap X1000). Inset shows HSIL cells.

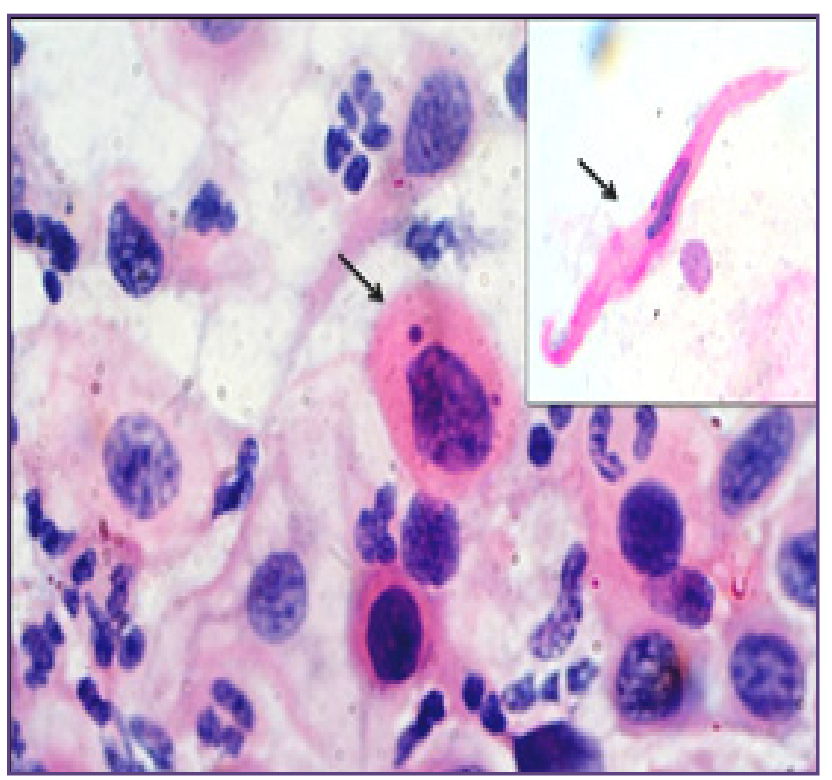

Fig. 2.Cervical smear of an invasive SCC case showing MN. Inset shows a tadpole cell with aMN(Pap X1000). 


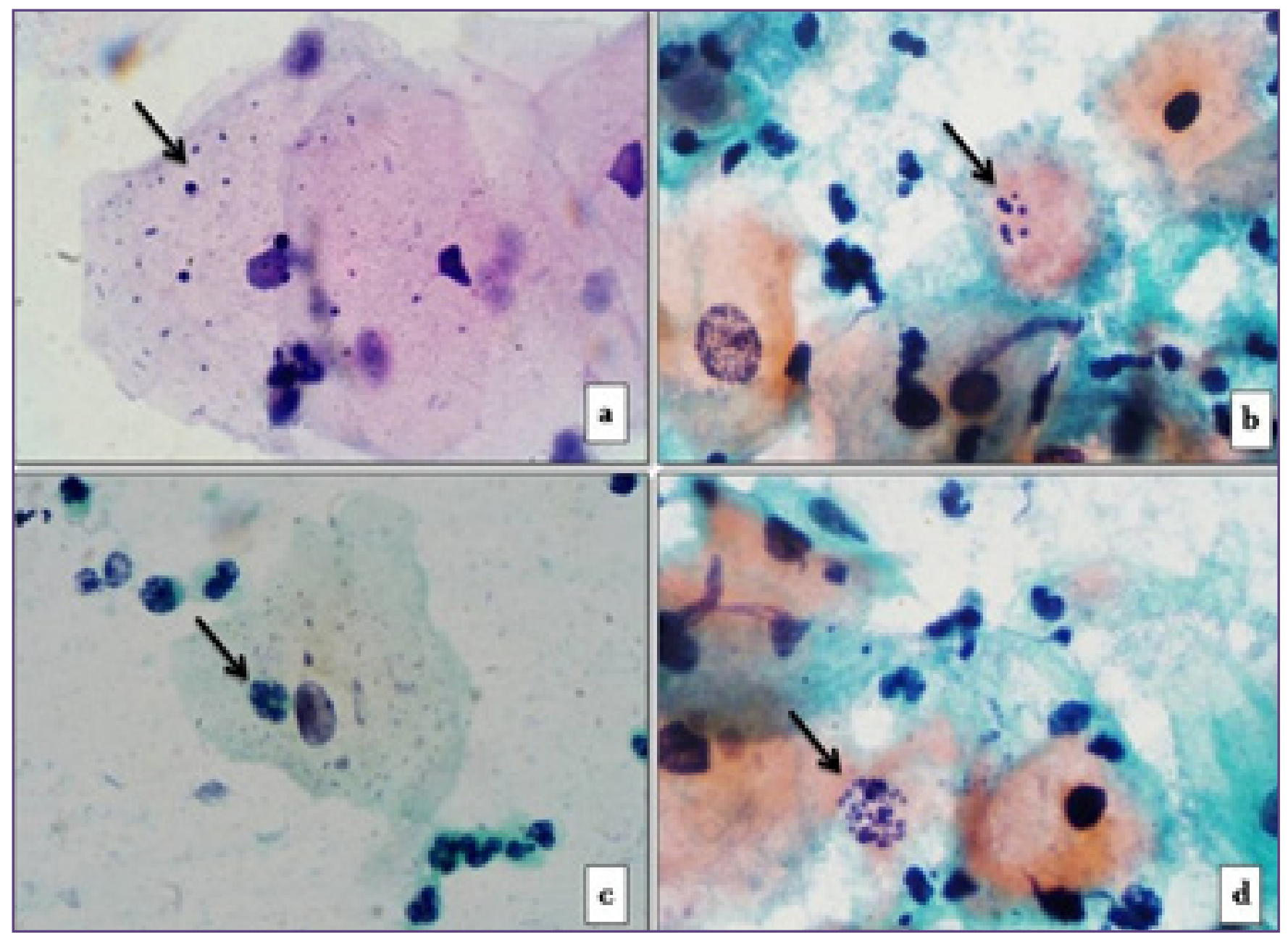

Fig. 3: Various mimics of MN. a. Multiple dot-likekerato-hyaline granules; b. A cell showing apoptotic bodies in a case of invasive SCC; c.Superimposed neutrophil and bacterial debris simulating micronucleus and d. Karyorrhexis in a case of invasive SCC (Pap x1000).

Though vaccination against HPV infection and periodical Papanicolaou cervical cytology screening are effective measures for preventing cervical cancer; there is a need for further improvement in the test in order to increase its sensitivity. ${ }^{[1]}$ Since HPV infection induces cytogenetic instability in cervix cells, it can be evaluated by means of $\mathrm{MN}$ assay. Human bio-monitoring studies have shown that $\mathrm{MN}$ assay in exfoliated cells is a site-specific biomarker of exposure to genotoxic agents and cancer risk. ${ }^{[12]}$. Among the various non- invasive early detection methods, MN formed in vivo can be used as a suitable biomonitoring approach for detection of increased cancer risk in man, because $>90 \%$ of all human cancers are of epithelial origin.

In the present study, we noted a gradual increase in MNscoreaccording to the severity of phenotypic changes. Ourfindings are in concordance with those of previous studies by Samanta et $\mathrm{al}^{[1]}$, Guzman et $\mathrm{al}^{[13]}$ and Leal

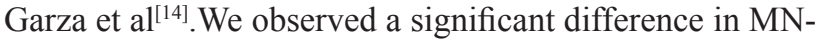
frequency between HSIL/ invasive SCC groups and other groups. ASC-H with reactive outcome had a significantly lower MN-frequency as compared to those with CIN outcome. However, the MN-score between HSIL and invasive SCC was statistically not significant. Samanta et $\mathrm{al}^{[1]}$ (2011) studied normal, inflammatory, ASCUS, ASC-H, LSIL and HSIL smears as separate groups and observed a statistically significant and gradually increasing $\mathrm{MN}$ score among these lesions.

Leal Garza(14) found that $\mathrm{MN}$-frequency in pap smear increases with increasing grade of cervical intraepithelial lesionsand there was a positive linear trend between the $\mathrm{MN}$ - frequency and increased cancer risk. Guzman(15) observed that $\mathrm{MN}$-frequency of more than one was more 
commonly found in LSIL (18\%), HSIL (33\%) and invasive carcinomas $(16 \%)$ as compared to ASCUS $(6 \%)$ and normal controls $(3 \%)$. However there was no significant difference in the MN-frequencies of ASCUS cases when compared with normal smears.

The wide variation in the $\mathrm{MN}$-frequency among the individuals within the same group are attributed to various confounding factors like lifestyle, environmental exposure, micronutrient deficiency, genetic makeup, base line MNfrequencies and chromosomal damage.(1)

The possible mechanisms(14) for progressive increase in MN in the cervical cancer patients are (1) the metabolic stress due to tumor growth, (2) the "clastogenic" product released by the tumor cells, (3) micronutrient deficiencies such as the folate and the vitamin B12 and (4) the presence of HPV.

So it is evident that the cytomorphology together with MNfrequency helps in discriminating cellular atypia due to reactive change versus dysplastic change. The study of MN in Pap smears increases the sensitivity and specificity of cervical smears which could have an impact in diagnostics and secondary prevention of cervical cancer.(16) Hence, the frequency of MN can be used as an additional criterion for assessing the cervical cancer risk.

Positive message from study: MN-scoring is a simple, non invasive and cost effective test, which can be performed on an easily collected cell sample. The processing and staining are less time consuming thanother test systems. The test can be performed in anyminimally equipped cytology laboratory. Though MN counting is time consuming and relatively tedious process with a need for diligent approach, it'sa worth while test in situations with genuine difficulty in distinguishing reactive versus ASC-H cases.

Limitations of study: Relatively less number of samples and exclusion of inflammatory smears are a minor limitation of our study. As LSIL cases generally do not undergo biopsy follow up, they were not included in the study.

Implication of this study: This simple test of detection of $\mathrm{MN}$ in conventional Papsmears can be used as a cost effective prognostic indicator during planning and validation of programs for cervical cancer screening, monitoring and prevention.

\section{Conclusion}

MN assay in conjunction with conventional Pap test can be utilized in screening cervical cancer and identifying women at risk. However, additional studies are needed to compare spontaneous and induced $\mathrm{MN}$ in cervical epithelial tissues, to standardize protocols and validate the induction of $\mathrm{MN}$ in epithelial tissues as biomarker of cancer risk.

\section{Refernces}

1. Samanta S, Dey P, Nijhawan R. Micronucleus in cervical intraepithelial lesions and carcinoma. Acta Cytol. 2011;55(1):42-7.

2. Delfino V, Casartelli G, Garzoglio B, Scala M, Mereu $\mathrm{P}$, Bonatti S, et al. Micronuclei and p53 accumulation in preneoplastic and malignant lesions of the head and neck. Mutagenesis. 2002;17(1):73-7.

3. Karaman A, Binici DN, Kabalar ME, Calikuşu Z. Micronucleus analysis in patients with colorectal adenocarcinoma and colorectal polyps. World J Gastroenterol WJG. 2008 ;14(44): 6835-9.

4. Aires GMA, Meireles JRC, Oliveira PC, Oliveira JL, Araújo EL, Pires BC, et al. Micronuclei as biomarkers for evaluating the risk of malignant transformation in the uterine cervix. Genet Mol Res GMR. 2011;10(3):1558-64.

5. Arora SK, Dey P, Saikia UN. Micronucleus in atypical urothelial cells. Diagn Cytopathol. 2010;38(11):811-3.

6. Fenech M, Holland N, Chang WP, Zeiger E, Bonassi S. The HUman MicroNucleus Project--An international collaborative study on the use of the micronucleus technique for measuring DNA damage in humans. Mutat Res. 1999;428(1-2):271-83.

7. Solomon D. The Bethesda system for reporting cervical cytology: definitions, criteria, and explanatory notes. New York: Springer; 2004.pp?

8. Manos MM, Kinney WK, Hurley LB, Sherman ME, ShiehNgai J, Kurman RJ, et al. Identifying women with cervical neoplasia: using human papillomavirus DNA testing for equivocal Papanicolaou results. JAMA J Am Med Assoc. 1999;281(17):1605-10.

9. Solomon D, Davey D, Kurman R, Moriarty A, O'Connor D, Prey M, et al. The 2001 Bethesda System: terminology for reporting results of cervical cytology. JAMA J Am Med Assoc. 2002;287(16):2114-9.

10. Saxena U, Sauvaget C, Sankaranarayanan R. Evidencebased screening, early diagnosis and treatment strategy of cervical cancer for national policy in low- resource countries: example of India. Asian Pac J Cancer Prev APJCP. 2012;13(4):1699-703.

11. Leyden WA, Manos MM, Geiger AM, Weinmann S, Mouchawar J, Bischoff K, et al. Cervical cancer in women with comprehensive health care access: attributable factors in the screening process. J Natl Cancer Inst. 2005;97(9):675-83.

12. Fenech M, Kirsch-Volders M, Natarajan AT, Surralles J, Crott JW, Parry J, et al. Molecular mechanisms of 
micronucleus, nucleoplasmic bridge and nuclear bud formation in mammalian and human cells. Mutagenesis. 2011;26(1):125-32.

13. Guzmán P, Sotelo-Regil RC, Mohar A, Gonsebatt ME. Positive correlation between the frequency of micronucleated cells and dysplasia in Papanicolaou smears. Environ Mol Mutagen. 2003;41(5):339-43.

14. Leal-Garza CH, Cerda-Flores RM, Leal-Elizondo E, CortésGutiérrez EI. Micronuclei in cervical smears and peripheral blood lymphocytes from women with and without cervical uterine cancer. Mutat Res. 2002;515(1-2):57-62.

15. Guzmán P, Sotelo-Regil RC, Mohar A, Gonsebatt ME. Positive correlation between the frequency of micronucleated cells and dysplasia in Papanicolaou smears. Environ Mol Mutagen. 2003;41(5):339-43.

16. Nersesyan AK. Possible role of the micronucleus assay in diagnostics and secondary prevention of cervix cancer: a minireview. T $\square$ Sitologii $\square$ a Genet. 2007;41(5):64-6.

*Corresponding author:

Suganya Kuppovi Reddy, JIPMER, Puducherry, India

Phone: +413 2296562

Email: dr. suganya.k@gmail.com

Date of Submission : 12.02.2017

Date of Acceptance : 11.06.2017

Financial or other Competing Interests: None. 